Journal of Engineering and Applied Sciences 13 (22): 9618-9626, 2018

ISSN: 1816-949X

(C) Medwell Journals, 2018

\title{
Preparation Nano Thin Film of Polyaniline/MWCNT and Study of Their Response to Some Organic Matter Fumes
}

\author{
Mohammed Hadi Shinen, Amina K. Murad, S.A.A. AlSaati, Amjed Mirza Oda, \\ Fouad Z. Razooqi and Abbas Hussein Mugheer \\ Department of Science, College of Basic Education, University of Babylon, P.O. Box 4 Hillah, Iraq
}

\begin{abstract}
A Quartz Crystal Microbalance (QCM) sensor coated with a thin film of PANI/MWCNT was used as a sensitive method for the determination of a number of following vapours: Acetic acid, Chloroform and Benzene. The detection was based on the absorption of the vapours of these organic compounds into the film by using gas cell chamber. The detection of these vapours can be obtained in part per million (ppm). The research includes the use of PANI/MWCNT films with different thickness (44, 62 and 92) nm where thickness of the PANI/MWCNT films was controlled by the spin coating process. Results show that increasing of sensitivity with increase of the concentration of injected analyte when the PANI/MWCNTfilm-coated QCM was exposed to the vapours of Acetic acid, Chloroform and Benzene. Best sensitivity to Chloroform and Benzene were obtained with film thicknesses $62 \mathrm{~nm}$ while in the case of Acetic acid best results were obtained with thickness of $92 \mathrm{~nm}$.
\end{abstract}

Key words: QCM, polyaniline, MWCNT, vapors, sensor, thickness

\section{INTRODUCTION}

What is a sensor: A device that receives and responds to a stimulus or signal. A sensor measure real world conditions, such as heat, gases, light and then converts this condition into analogue or digital representation of data, e.g., QCM Quartz Crystal Microbalance Sensors and MOS Metal Oxide Semiconductor sensors (Sauerbrey, 1959; Mecea, 2005).

QCM-Quartz Crystal Microbalance sensors: The typical structure of a QCM sensor is shown in the Fig. 1. The quartz has the shape of a disc with the two gold electrodes on the two opposite surfaces. On top of one of the metallization an active layer, usually polymeric is deposed. These sensors are very easy to realize inexpensive, they work at ambient temperature, so they, do not require a heating structure have a quite good resolution. Nevertheless the stability of the polymers is in general lower than that of metal oxides and these sensors suffers of aging as shown in Fig. 1 a, b (Mecea, 2005).

In $\mathrm{AC}$ signal is applied between the electrodes resulting in a shear deformation wave across the crystal. Due to the piezoelectric properties of the quartz, it can be electrically excited into a number of resonant modes with maximum displacement occurring at the crystal faces, the property of maximum displacement at the crystal faces in the Thickness Shear Mode (TSM) makes this mode of excitation extremely sensitive to surface mass accumulation (Ballatine et al., 1997; Narumi et al., 2009).

In a generic QCM based measurement system the quartz sensors are placed in oscillator circuits. The value of the quartz resonance frequency depends both on the characteristics of the crystal and on those of the sensitive layer.The crystal has a characteristic resonance frequency, usually in the $\mathrm{MHz}$ range whereas the film deposition on the bulk causes a shift of the resonance frequency up to a few hundreds of part per million (ppm) (Mecea, 2005).

The gas is injected (in most cases with a flow controlled) directly on the sensitive layer surface. When the gas gets into contact with the layer, the molecules are sorbed in the sensitive film. This fact causes a mass variation of the sensor and consequently a shift of the resonance frequency. An estimate of the initial gas concentration is the result of the measure of this frequency shift (Narumi et al., 2009).

Thus, the response of the coated QCM sensors is based on the different sorption and diffusion characteristics of the individual compounds in the polymeric coating. Different compounds take different

Corresponding Author: Mohammed Hadi Shinen, Department of Science, College of Basic Education, University of Babylon, P.O. Box 4 Hillah, Iraq 


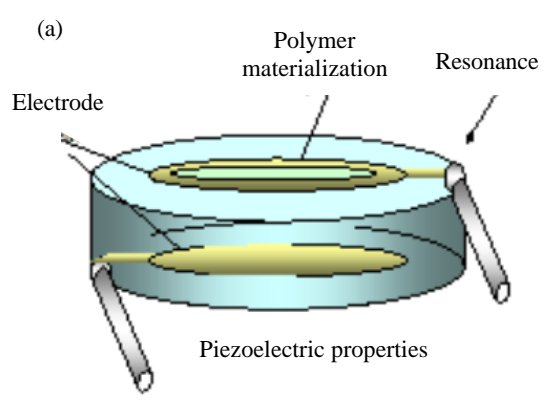

(b)

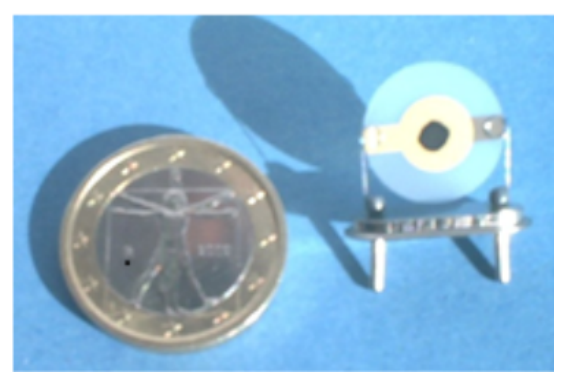

Fig. 1: a) Source and b) QCM structure

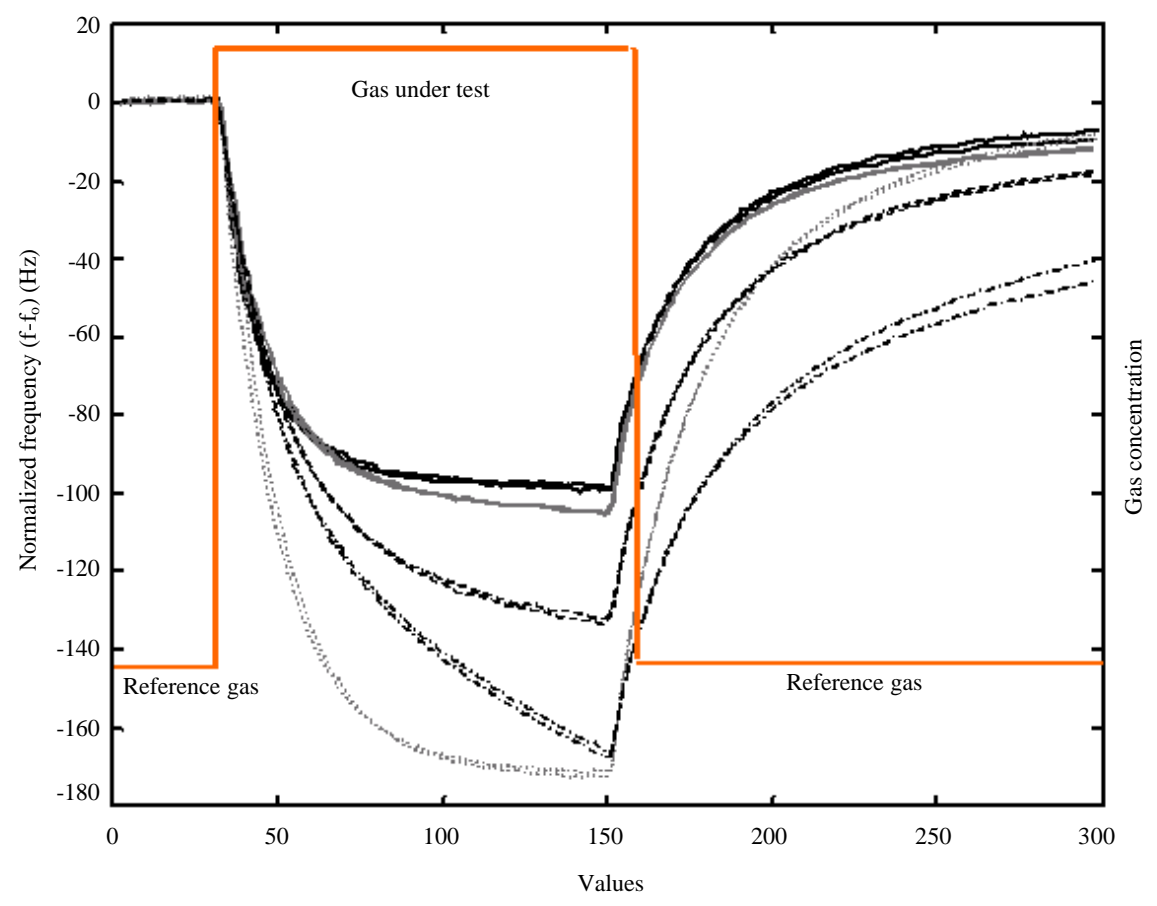

Fig. 2: Transient sensor response of a QCM sensor to 5 different gas mixtures

times to reach steady-state in the polymer (Hussien and Shinen, 2013). The response of typically QCM is shown in Fig. 1-3 (Hu et al., 2009).

Hence, the selectivity of these sensors can be improved by measuring also the dynamic characteristics. This can be achieved by an electronic system able to track the transient behavior. In this context our activity research is related to the measurement and the study of the transient response of QCM sensors (Sauerbrey, 1959).

The chemical structure and physical properties of polymeric coatings and the nature of interaction between polymer coatings and vapour molecules determine the selectivity, sensitivity, signal kinetics and reversibility of the sensors (McGill et al., 2000).
Conducting polymers such as Polyaniline (PANI), polypyrrole and polythiophene have been widely investigated as effective materials for chemical sensors (Shinen et al., 2014).

The advantages of conducting polymers compared to inorganic materials used until now are their diversity, their easy synthesis and particularly, their sensitivity at room temperature (Wang et al., 2006). Furthermore, conducting polymers have good mechanical properties which allow a facile fabrication of sensors. As a result, more and more attentions have been paid to the sensors fabricated from conducting polymers and a lot of related articles were published, for example, polyaniline was found to be a better choice for gases such as ammonia because of its higher sensitivity, reversible response andshorter response time (Wang et al., 2006). 


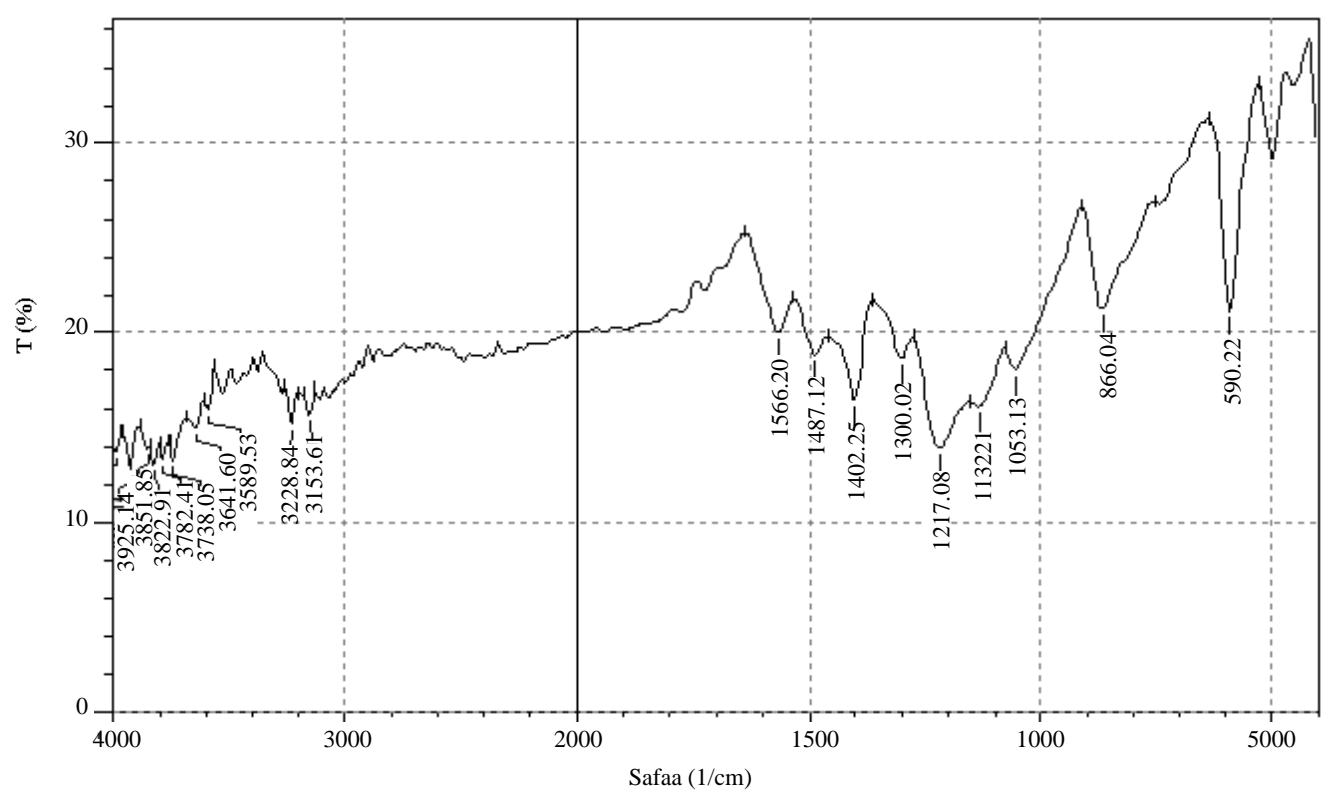

Fig. 3: FTIR for pure polyaniline

studied the sensitivity, short-term reproducibility, stability and response time properties of $\mathrm{ZnO}$ nanowires-modified quartz crystal as a gas-detecting sensor. Shinen et al. (2014) used Quartz Crystal Microbalance (QCM) sensor, coated with a thin film of polyaniline Emeraldine Base (EB) as a sensitive method for the determination of a number of aliphatic chlorinated hydrocarbons such as carbon tetrachloride, chloroform, dichloromethane, 1, 2dichloroethane vapours. Polyaniline-modified Quartz Crystal Microbalance (QCM) sensor was obtained through immobilizing the polyaniline film on the silver electrode surface of quartz crystal resonator by an electrochemical method and the sensor was studied for detecting the formic acid gas of different concentrations (Yan et al., 2012; Shinen et al., 2016) and a lot of related articles were published, for example, polyaniline was found to be a better choice for gases such as ethanol, propanol, hexane and benzene because of its higher sensitivity, reversible response and shorter response time (Hussien and Shinen, 2013). The aim of the present research was to employ different thickness PANI film coated QCM as a sensor for the following vapours: acetic acid, chloroform and benzene.

\section{MATERIALS AND METHODS}

Synthesis of polyaniline: Polyaniline is prepared to take the $6 \mathrm{mmol}$ of aniline in $30 \mathrm{~mL}$ of hydrochloric acid concentration $1 \mathrm{M}$ and placed on electromagnetic stirrer for $1 \mathrm{~h}$ and then cooled to a temperature of $-5^{\circ} \mathrm{C}$ for $2 \mathrm{~h}$. After that another solution is prepared consisting of 6 mmol of ammonium peroxidisulphate and added mechanism $30 \mathrm{~mL}$ of hydrochloric acid concentration $1 \mathrm{M}$ and placed on board for a period of $1 \mathrm{~h}$. Then two solutions are mixed well gradual for $2 \mathrm{~h}$ to precipitate urinary polyaniline. Then added a very small amount of sodium hydroxide concentration 1 Murlara into the mix to precipitate minutes polycarbonate polyaniline. Then the solution is washed with distilled water a number of times and then with ethanol and then dried product for a day by a rarefied drying under pressure when the temperature is freezing after getting urinary polyaniline (Pawar et al., 2009).

Synthesis of thin film: Different thickness of PANI/MWCNT films were deposited on both sides of Quartz Crystal Microbalance (QCM) by using spin-coating. PANI films obtained were (44, 62 and 92) nm and thickness measurements were achieved by using ellipsometry. Gas cell chamber (controlled by software) was used to measure the sensitivity of QCM when exposed to different analysts of ofAcetic acid, Chloroform and benzene. The QCM was exposed to air after the absorption of each analyte. The backshift of the crystal frequency to its initial value was taken as an indication of full desorption. All measurements were carried out at room temperature. Sensitivity is defined as the ability of a sensor to produce a signal when low concentrations of a target analyte are present. The larger the signal, the more sensitive a sensor is. The sensitivity of a sensing material is defined as the concentration of analytesorbed onto the sensing material divided by the total concentration of the analyte (Stewart, 2011). 
QCM sensing membranes and film deposition method: For making the QCM to work as a chemical sensors it is coated with a membrane suitable for the target analyze for which it is planned to do. Spin coating method is selected and used in our process, among different methods that can be used for coating such as spray coating, spin coating and Langmuir Blodgett. Spin coating method is selected on the basis of the easy accessibility of the user in the research and the equipments and the apparatus which are available in the laboratory (Hussien and Shinen, 2013).

It is important to notice the physical structure of the QCM. It should be clean without any defects to the gold discs of the QCM, there should not be any poor contact between the electrodes and the quartz which can be easily seen by the human eyes. All crystals were rinsed with thoroughly using acetone and dried with a stream of pure nitrogen before the initial measurement of the uncoated QCM is taken. To keep the QCM without any damage, it is handled with high care, users wear gloves, they use tweezers and puckers to operate the QCM in the process to avoid and damage contamination. All this process is carried out in a well clean room with a good atmosphere (Shinen et al., 2016)

\section{RESULTS AND DISCUSSION}

FTIR: Figure 3 illustrates the frequency of the bonds of effective aggregates sites for pure polyaniline film. The emergence of a summit in the $\left(3153.61 \mathrm{~cm}^{-1}\right)$, this indicates the presence of $\mathrm{C}-\mathrm{H}$ Aromatic is an important feature of the reference to the polyaniline, the emergence of values between $\left(1487-1566.20 \mathrm{~cm}^{-1}\right)$ these values back to the presence of Yle group $\mathrm{C}=\mathrm{C}$ Aromatic ringed in the polyaniline. When vaccination with carbon nano-tube as in Fig. 4 are seeing the emergence of value at $3210 \mathrm{~cm}^{-1}$ and this is clear evidence of a $\mathrm{C}-\mathrm{H}$ aromatic attributable to polyaniline this corresponds to approximately the same value that appeared when you reference the value of which (3153.61 $\left.\mathrm{cm}^{-1}\right)$ as in Fig. 2. The emergence of value at $\left(2958.80 \mathrm{~cm}^{-1}\right)$ this returned to $\mathrm{CH}_{3}$ in carbon nanotubes as referred to in the basic material as in Fig. 5, the emergence of value at $2927.94 \mathrm{~cm}^{-1}$ returning to Yle group $\mathrm{CH}_{2}$ in carbon nanotubes and also, the emergence of value at $\left(2779.42 \mathrm{~cm}^{-1}\right)$ returning stretchable $(\mathrm{CH})$ in carbon nanotubes, he emergence of values between (11635.64- $1486 \mathrm{~cm}^{-1}$ ) evidence of a $\mathrm{C}=\mathrm{C}$ aromatic attributable to polyaniline.

These results indicated to the presence of each of the polyaniline and carbon nano-tube which indicates to their presence together.

XRD: Figure 6 which represents the X-ray diffraction of pure polyaniline, appears broad summit at (250) which shows that the poly Aniline is (Amorphous) while Fig. 7 represents X-ray diffraction of the poly-aniline doped by Carbon nanotubes with ratio $1 \%$ where notes the appearance of two peaks at $\left(5^{\circ}-30^{\circ}\right)$. An angle $\left(5^{\circ}\right)$ shows the diffraction for the parallel levels in the polymeric chain an angle $\left(30^{\circ}\right)$ shows the diffraction of the levels orthogonal.

AFM: The results of the tests (AFM) of the nano films for pure (PANI) and doped with different ratio of (MWCNTS) films which prepared by spin coating which showed a uniform granular surface morphology as in Fig. 8 and 9.

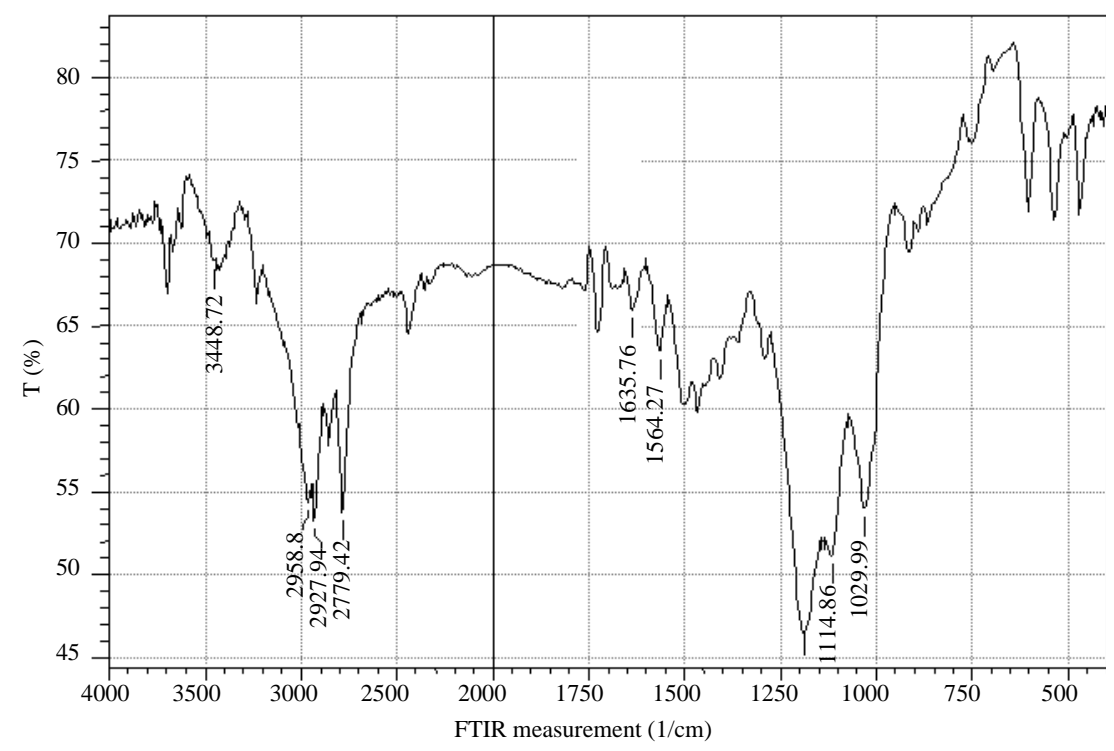

Fig. 4: FTIR for polyaniline/MWCNTS 


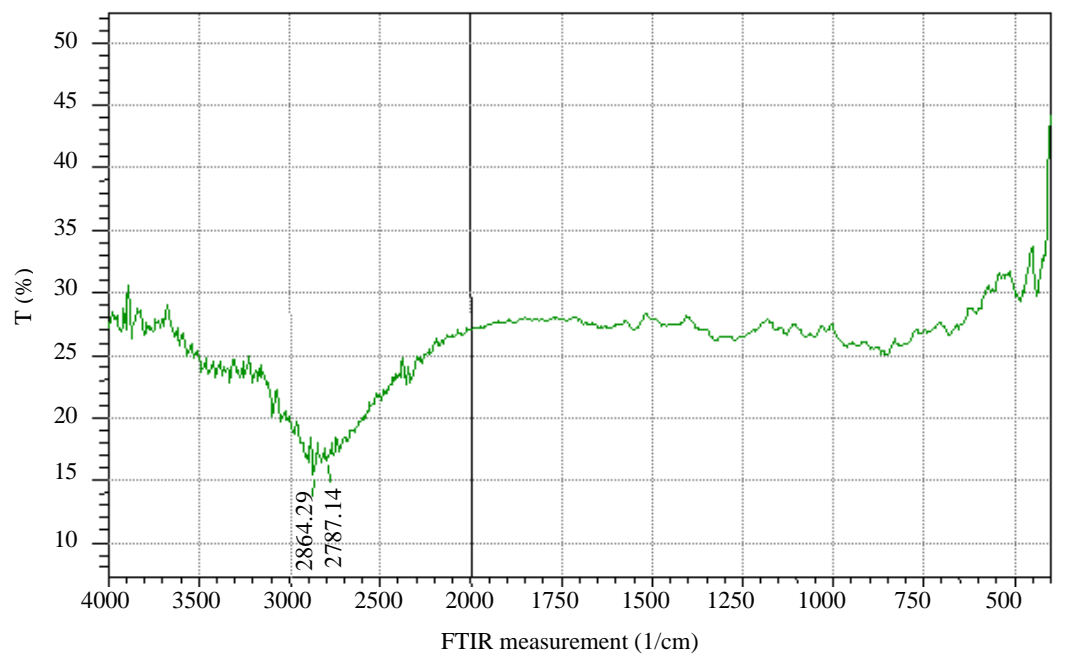

Fig. 5: FTIR for carbon nanotubes (MWCNTS)

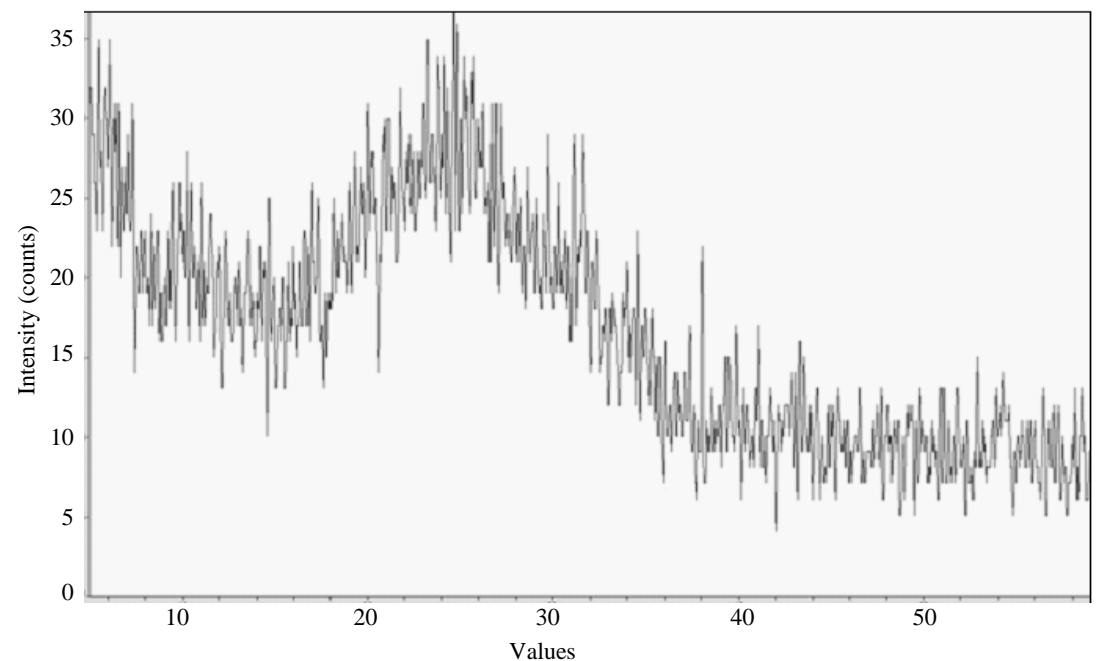

Fig. 6: X-ray for pure polyaniline

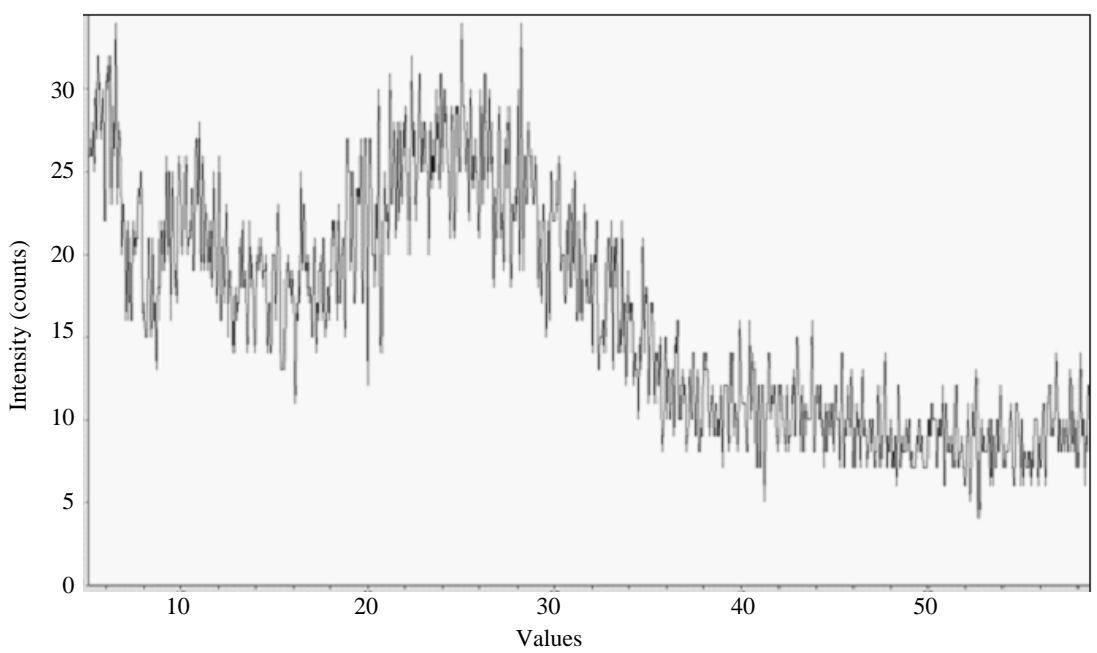

Fig. 7: X-ray forpolyaniline/MWCNTS 


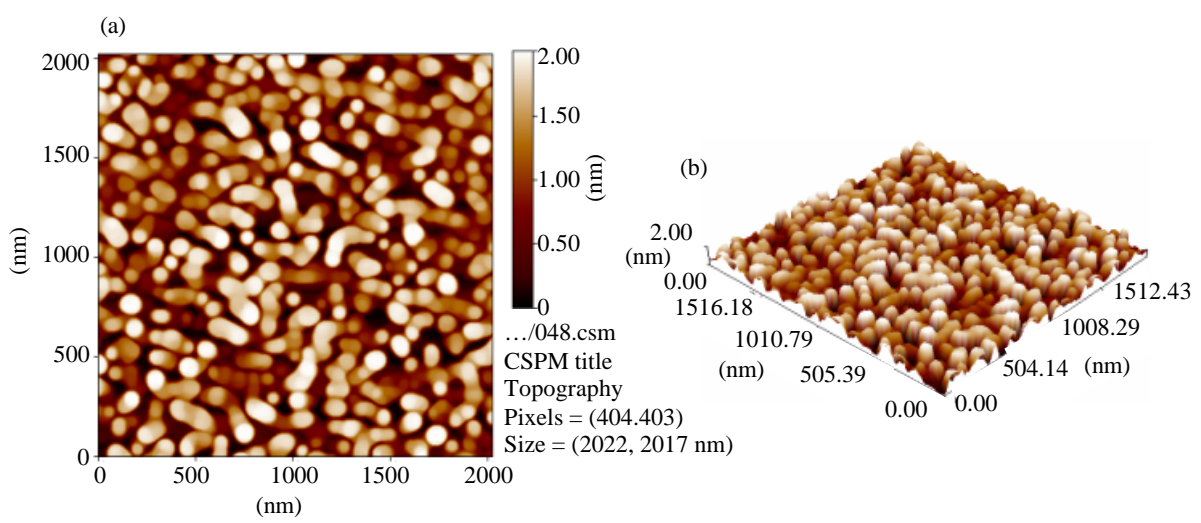

Fig. 8: AFM images of pure PANI nano films for (a) 2-D and (b) 3-D

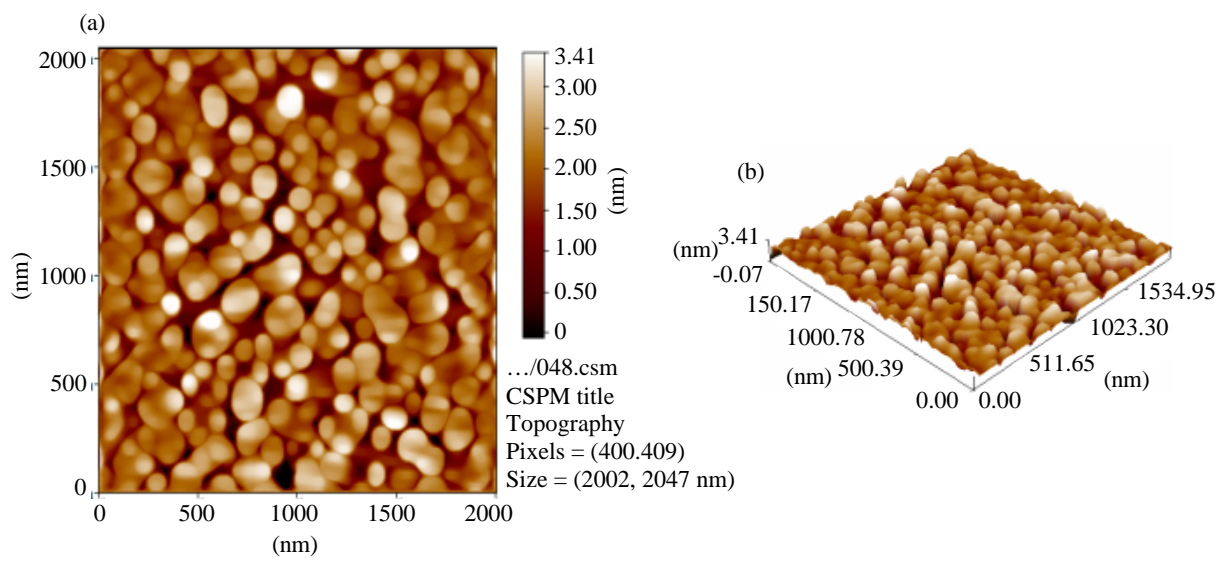

Fig. 9: AFM images of PANI/ MWCNTS for (a) 2-D and (b) 3-D

Where we note that the roughness increased with increasing the ratio of doped. As well The Root Mean Sequare (RMS) increased with increasing of ratio of doped, the average grain diameter also It exhibits the same behavior. This is consistent with the findings of the researchers (Abdullah et al., 2015; Ziadan and Inayh, 2016).

QCM sensor: Figure 10-12 show an increase and decrease in sensitivity according to the amount of vapor injected $(5-10 \mu)$ of the three nano thin film of PANI/MWCNT coated QCM was exposed to the vapours of Acetic acid, Chloroform and Benzene. This is expected, since, when more vapor molecules are provided in the test atmosphere, more molecules would be absorbed into the PANI/MWCNT coating on the QCM. After each addition of vapor concentration, the frequency of the crystal was back shifted to its initial value by drying the electrode using air which indicates full desorption of vapor from the electrode surface. This behaviour confirms that the sensing interaction between the imine and amine sites of
PANI/MWCNT chains coating and vapor is a physical absorption through a dipole/dipole interaction or hydrogen bonding. The presence of hydrogen attached to an electronegative nitrogen in all of the polymers allows for hydrogen bonding (Stewart, 2011). Figure 10 shows increasing of sensitivity with increase of the concentration of injected analyte of Acetic acid. The increase in resistivity of the sensor on exposure to Acetic acid is thought to be caused by an interaction via dipole/dipole moment of Acetic acid molecules and the nitrogen atoms of PANI/MWCNT, leading to the hindrance in conformational rearrangements of the macromolecules that impedes electron delocalization and charge transport through the polymer chain (Narumi et al., 2009). Best sensitivity was obtained with film thicknesses $62 \mathrm{~nm}$ at high and low concentration of Acetic acid. So, it is found that the PANI/MWCNT thickness would affect the sensitivity of the sensor. This can be assigned to the increased active sites of the PANI/MWCNT as the film thickness increases. Therefore, PANI/MWCNT of 62 

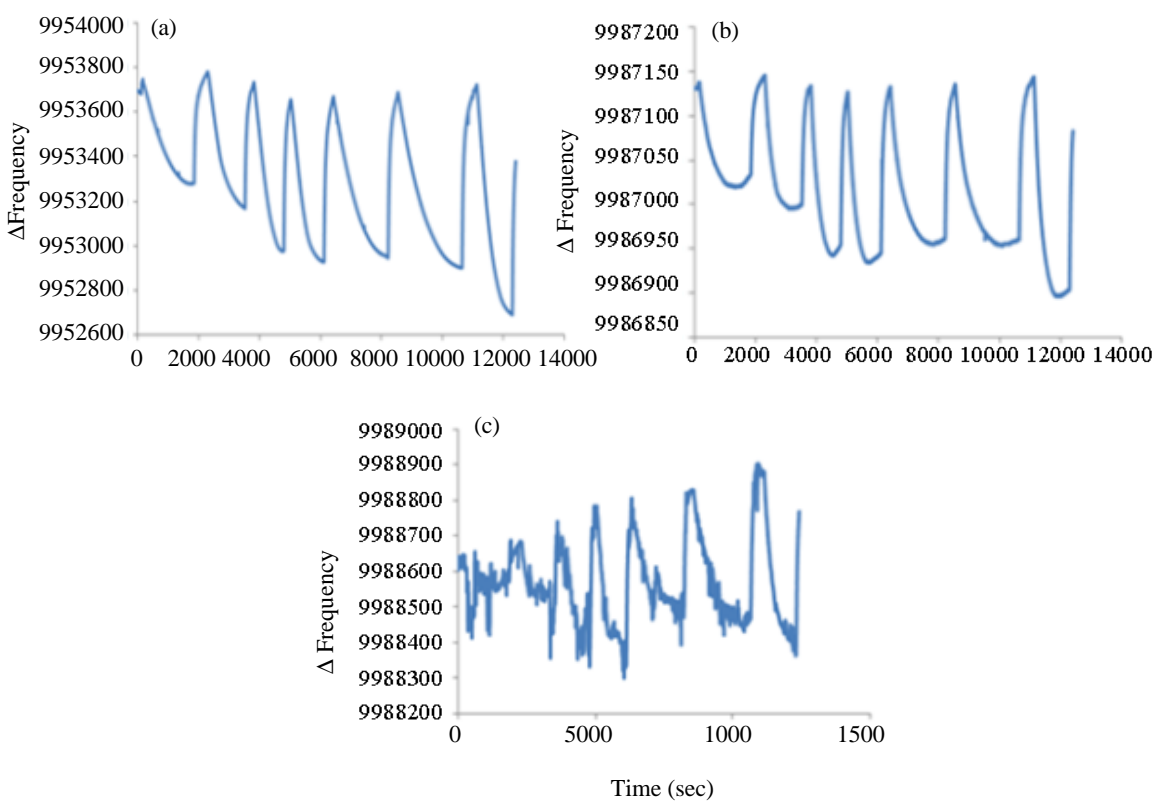

Fig. 10: Sensitivity of QCM Coated with different thickness of PANI/MWCNT exposed to acetic acid: a) $44 \mathrm{~nm}$; b) 62 $\mathrm{nm}$ and c) $92 \mathrm{~nm}$
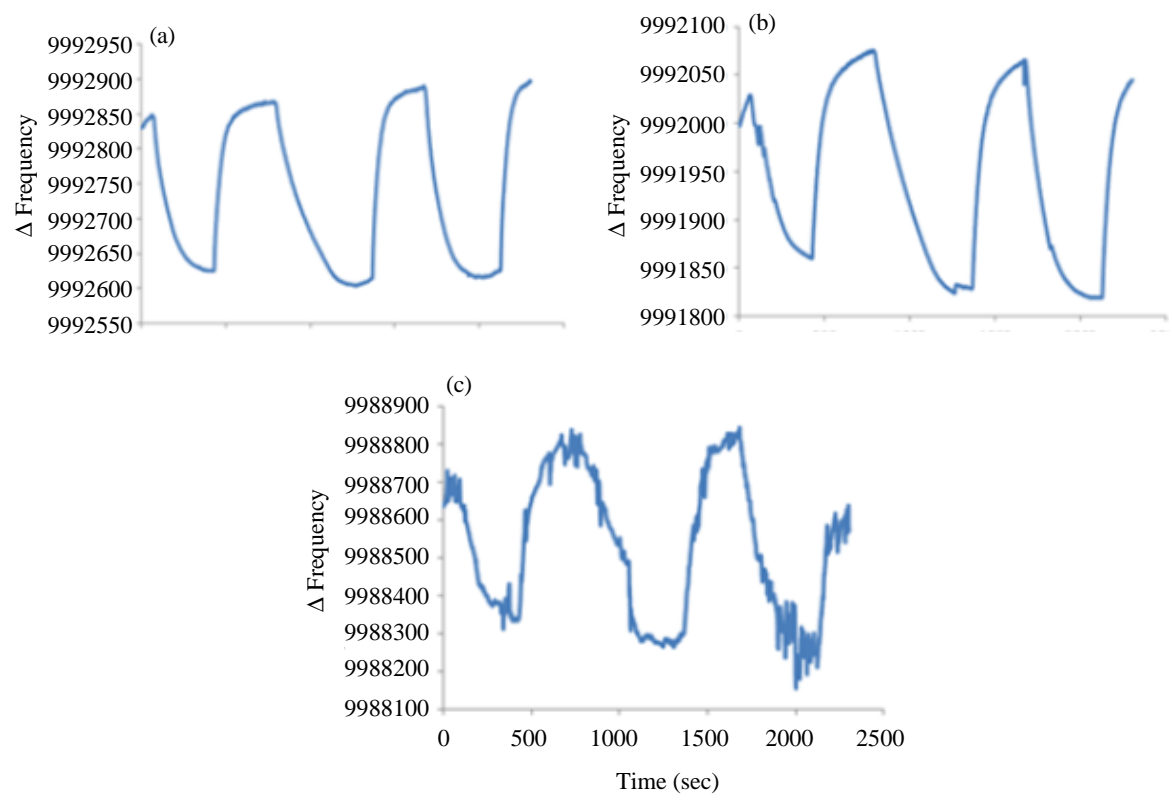

Fig. 11: Sensitivity of QCM Coated with different thickness of PANI/MWCNT exposed to chloroform: a) $44 \mathrm{~nm}$; b) 62 $\mathrm{nm}$ and c) $92 \mathrm{~nm}$

nm considered suitable thickness to create active sites and but Acetic acid molecules only physically absorb on the PANI/MWCNT surface. On the other hand it was found that thickness of 44 and $92 \mathrm{~nm}$ gave low sensitivity at high and low concentration to Acetic acid shown in Fig. 10 due to poor physically absorb on the PANI/MWCNT surface.
Figure 11 illustrates the effect of PANI/MWCNT film thickness on sensitivity of QCM with increase of the concentration of injected analyte of Chloroform. Also, it can be seen that with increase in analyte concentration the sensitivity increases which could be attributed to the interaction via. dipole/dipole moment of Chloroform molecules molecules and the nitrogen atoms of 


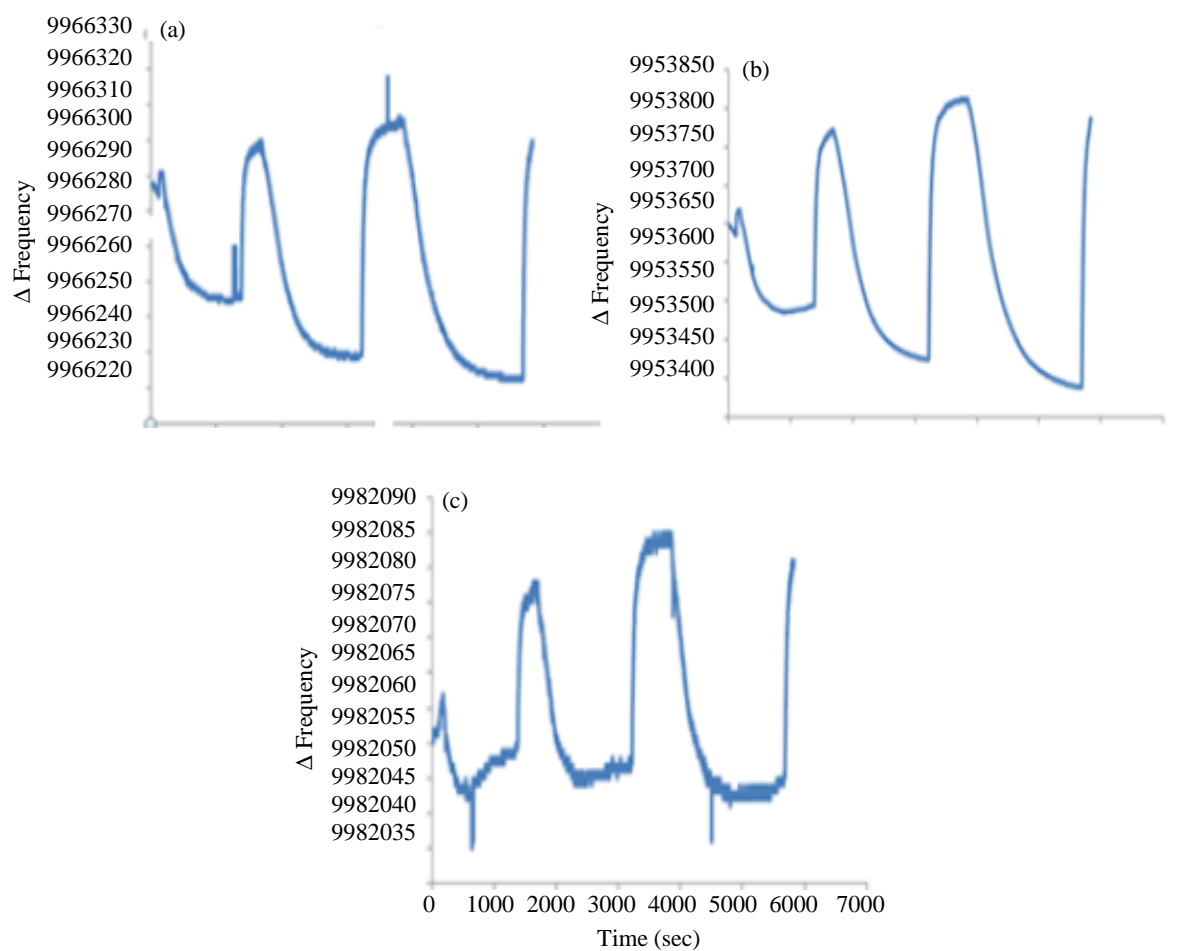

Fig. 12: Sensitivity of QCM Coated with different thickness of PANI/MWCNT exposed to Benzene: a) $44 \mathrm{~nm}$; b) $62 \mathrm{~nm}$ and c) $92 \mathrm{~nm}$

PANI/MWCNT. As shown in Fig. 11, best results were obtained with thickness of 62 and $44 \mathrm{~nm}$. By comparison Fig. 10 and 11, it can be seen that QCM with PANI/MWCNT film of thickness $62 \mathrm{~nm}$ has more sensitivity to Chloroform because more physical absorptiontake place. This is because low molecular weight alcohols vapors decrease the conductivity of the PANI/MWCNT faster than higher molecular weight alcohols. These results are in agreement with the literature. For example, Sauerbrey (1959), Ballantine et al. (1997), Hussien and Shinen (2013) observed a much slower response for ethanol vapor when compared to methanol vapor. It was interpreted that the electrons in the PANI were much more tightly bound in the presence of the low molecular weight alcohol.

Figure 12 demonstrates behavior of QCM towards increase of the concentration of injected analyte of Benzene is non-polar molecular. This would then indicate that polarity is not the only factor ingenerating a response on the nanosensors (Yan et al., 2012). Elucidation of the exact mechanisms would require extensive research with a variety of substances and possible development of structure activity relationships. Also, it can be seen that best sensitivity to Benzene was obtained with PANI/MWCNT film thicknesses $62 \mathrm{~nm}$.

\section{CONCLUSION}

The summarized results from this research are the following:

- It is found through a study that these polymers possess random installation

- The addition of carbon nano-tube does not change the installation of the polymer but are attending together

- All films are uniform granular surface morphology

\section{REFERENCES}

Abdullah, E.T., R.S. Ahmed, S.M. Hassan and A.N. Naje, 2015. Synthesis and characterization of PANI and polyaniline/multi walled carbon nanotube composite. Intl. J. Appl. Innovation Eng. Manage., 4: 130-134.

Ballantine, D.S., S.J. Martin, A.J. Ricco, R.M. White and G.C. Frye, et al., 1997. Acoustic Wave Sensors: Theory, Design and Physico-Chemical Applications. Academic Press, Cambridge, Massachusetts, USA., ISBN:9780120774609, Pages: 436.

Hu, G., J.A. Heitmann Jr. and O.J. Rojas, 2009. In situ monitoring of cellulase activity by microgravimetry with a quartz crystal microbalance. J. Phys. Chem. B, 113: 14761-14768. 
Hussien, H.M. and M.H. Shinen, 2013. Study the sensitivity of Quartz Crystal Microbalance (QCM) sensor coated with different thickness of polyaniline for determination vapours of ethanol, propanol, hexane and benzene. Chem. Mater. Res., 3: 61-65.

McGill, R.A., T.E. Mlsna, R. Chung, V.K. Nguyen and J. Stepnowski, 2000. The design of functionalized silicone polymers for chemical sensor detection of nitroaromatic compounds. Sens. Actuators B Chem., 65: 5-9.

Mecea, V.M., 2005. From quartz crystal microbalance to fundamental principles of mass measurements. Anal. Lett., 38: 753-767.

Narumi, K., A. Asakura, T. Fukuda and F. Arai, 2009. Compact force sensor using AT-cut quartz crystal resonator supported by novel retention mechanism. J. Rob. Mechatron., 21: 260-266.

Pawar, S.G., S.L. Patil, A.T. Mane, B.T. Raut and V.B. Patil, 2009. Growth, characterization and gas sensing properties of polyaniline thin films. Arch. Appl. Sci. Res., 1: 109-114.

Sauerbrey, G., 1959. [Use of quartz crystals for weighing thin layers and for weighing (In German)]. Z. Fur Phys., 155: 206-222.
Shinen, M.H., F.O. Essa and A.S. Naji, 2014. Study the sensitivity of Quartz Crystal Microbalance (QCM) sensor coated with different thickness of polyaniline for determination vapors of ether, chloroform, carbon tetrachloride and ethyl acetate. Chem. Mater. Res., 6: 7-12.

Shinen, M.H., R. Mahmmod and S.S.A. Alabas, 2016. Preparation of thin films from conductive polymer PANI and study of their applications as a gas sensor. Intl. J. Chem. Tech. Res., 9: 749-753.

Stewart, K.M.E., 2011. Doped polyaniline for gas sensors for the detection of formaldehyde. Master's Thesis, University of Waterloo, Waterloo, Ontario.

Wang, X., J. Zhang and Z. Zhu, 2006. Ammonia sensing characteristics of $\mathrm{ZnO}$ nanowires studied by quartz crystal microbalance. Appl. Surf. Sci., 252: 2404-2411.

Yan, Y., D. Lu, H. Zhou, H. Hou and T. Zhang et al., 2012. Polyaniline-modified quartz crystal microbalance sensor for detection of formic acid gas. Water, Air Soil Pollut., 223: 1275-1280.

Ziadan, K.M. and H.H. Inayh, 2016. Preparation and characterization of nanocompocite conducting polymers (PANI-DBSA/MWNCT). Chem. Mater. Res., 8: 35-43. 